\title{
Modelo estadístico en series temporales para la predicción de la temperatura de gas de tragante del horno alto $^{(\bullet)}$
}

\author{
A. Martínez ${ }^{(*)}$, I. Rieiro ${ }^{(* *)}$, A. Formoso ${ }^{(*)}$ y A. Cores ${ }^{(*)}$
}

Resumen En el presente trabajo, a través de técnicas poco usadas en el campo siderúrgico, se establece un criterio para definir el comportamiento en el tiempo de variables de operación significativas del horno alto. Para ello, se ha desarrollado un modelo autorregresivo en series temporales que permite una adecuada predicción. La metodología ha consistido en utilizar las ecuaciones de Yule-Walker y el test de Akaike para establecer y elegir el mejor modelo, y el análisis de la función de autocorrelación para comprobarlo.

Palabras clave: Análisis de señal. Modẹlización. Temperatura de gas en tragante. Modelos autorregresivos. Modelización del horno alto.

\section{Time series statistical model to relate blast furnace operation variables}

\begin{abstract}
This work, through techniques seldom used in the ironmaking field, establishes a criterion to define the behaviour along the time of significant operating variables of the blast furnace. A time series autoregressive model has been developed, achieving with it an adequate forecasting level. The methodology used has been the Yule-Walker equations to establish the model, the Akaike test to choose the best one and the autocorrelation function analysis to check it.
\end{abstract}

Keywords: Signal analysis. Sample modelling. Top gas temperature. Auto regressive models. Blast furnace modelling.

\section{INTRODUCCIÓN}

El control de la operación del horno alto depende, en gran parte, de la calidad física y química de los componentes de la carga, formada fundamentalmente por coque $\left(\begin{array}{lll}1 & \text { y }\end{array}\right)$ y por aglomerados de minerales de hierro (3). Se han realizado numerosos estudios sobre la calidad de los aglomerados que forman la carga del horno, siendo los más utilizados los pelets (4) y los sinterizados (5 y 6). Además de la calidad de los componentes de la carga, es impor-

(•) Trabajo recibido el día 5 de julio de 1994.

(*) Centro Nacional de Investigaciones Metalúrgicas (CSIC). Avda. de Gregorio del Amo, 8. 28040-Madrid (España).

(**) Ministerio de Educación y Ciencia (MEC). Avda. Vascongadas, s/núm. 28903-Getafe. Madrid (España). tante el control y regulación del horno mediante la aplicación de adecuados modelos matemáticos.

Generalmente, los modelos matemáticos del horno alto se han basado en una descripción sistemática de sus procesos internos (7). Desde este punto de vista, son modelos analíticos y descriptivos, y necesitan un complejo equipo de medición. En la mayoría de los casos, ha sido necesario utilizar técnicas que afectan al medio a ensayar, destructivas incluso -como las disecciones de hornos realizadas en Japón (8)-, para conseguir una descripción formal de los fenómenos que ocurren en el interior del horno.

Se han usado técnicas muy diferentes: sondas verticales u horizontales, medidas de conductividad y de presión, etc., pero el instrumento principal utilizado en la mayoría de los hornos es el analizador continuo de gas en tragante, concretamente, el análisis de $\mathrm{CO}, \mathrm{CO}_{2}, \mathrm{H}_{2}$ y $\mathrm{N}_{2}$. 
Usando este dispositivo, conjuntamente con la información suministrada por otros medios, se han recogido datos fiables de temperatura, presión, composición y otros parámetros del gas en tragante que han hecho posible establecer diferentes modelos, algunos de los cuales son de gran eficiencia en el control del horno.

Por otro lado, es bien conocido que el horno alto es un sistema muy inercial, significando con ello que cualquier acción tendente a modificar su comportamiento tiene efecto con un retraso significativo. Este hecho dificulta enormemente el control del horno.

Los modelos de horno son descriptivos precisamente porque lo hacen de la forma en la que el horno está funcionando en el momento en el que se toman las medidas. Su capacidad de descripción del comportamiento futuro del mismo se basa, generalmente, en una extrapolación de los datos obtenidos.

El modelo desarrollado en el presente trabajo, que es parte de uno más amplio de modelización de la zona cohesiva (9), es más predictivo que descriptivo, ya que está basado en el análisis estadístico de series temporales de temperaturas, con el propósito de establecer una hipótesis sobre el funcionamiento futuro del horno. Establece la posibilidad de utilizar técnicas estadísticas para describir los procesos, usando una aproximación "sistémica" a la manera de Forrester (10), considerando el horno en cierto sentido como una "caja negra", haciendo abstracción de muchos de sus procesos internos, y realizando un estudio del comportamiento en el tiempo de las variables más significativas.

La técnica utilizada es la modelización autorregresiva de series temporales (modelos AR), que es bien conocida en otras áreas científicas pero escasamente utilizada en el campo siderúrgico.

\section{PARTE EXPERIMENTAL}

La variable elegida en este artículo como ejemplo de aplicación de esta técnica matemática es la temperatura de gas en tragante (TGT), reconocida habitualmente como de gran significación para conocer la marcha del horno.

Se han usado varios conjuntos de 240 valores de la TGT para la construcción de los modelos. Dichos valores se han obtenido en el horno núm. 3 de ENSIDESA (Avilés), durante períodos de $24 \mathrm{~h}$, efectuando las medidas a intervalos de $6 \mathrm{~min}$. En el presente trabajo se presenta el análisis en detalle de uno de dichos conjuntos. La figura 1 muestra una representación gráfica para este conjunto de valores.

El número relativamente pequeño de muestras hace imposible la utilización de la transformada rápida de Fourier. El modelo matemático básico aquí usado es el autorregresivo, que permite anali-

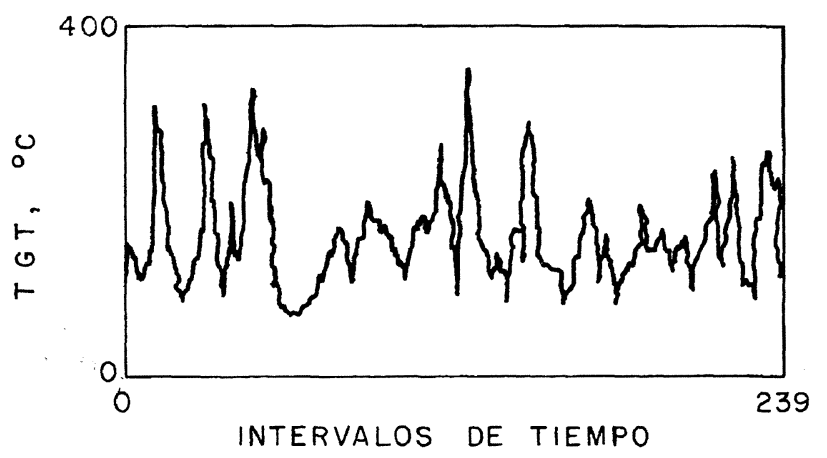

FIG. 1.- Perfil de la TGT, ${ }^{\circ} \mathrm{C}$. El período representado es de $24 \mathrm{~h}$ a intervalos de $6 \mathrm{~min}$.

\section{FIG. 1.- Profile of the $T G T,{ }^{\circ} \mathrm{C}$. The represented period is $24 \mathrm{~h}$, with 6 min intervale.}

zar muestras pequeñas (11). Dicho modelo viene definido por la ecuación:

$$
\operatorname{TGT}(t)=\sum_{\mathrm{k}=1}^{\mathrm{k}=\mathrm{p}} a(k) \cdot \operatorname{TGT}(t-k)+v(t)
$$

donde:

TGT $(t)=$ Temperatura de gas en tragante, ${ }^{\circ} \mathrm{C}$

$t=$ Tiempo, $\min$

$\mathrm{k}=$ Tiempo de retardo

$\mathrm{p}=$ Orden del modelo

$a(k)=$ Parámetros del modelo

$v(t)=$ Señal del tipo de ruido blanco

Para el modelo mencionado, se ha elegido un tiempo de demora de 20, significando que cada valor de la señal se ha relacionado con los precedentes en pasos que van desde 0 a 20 . Este proceso proporciona el valor de la función de autocorrelación, que describe el comportamiento en el tiempo de la señal completa (12). Los métodos usados para calcular los valores de los parámetros, incluyendo las ecuaciones de Yule-Walker, han sido los que usualmente se describen en libros de consulta (13).

El desarrollo del modelo tiene tres aspectos fundamentales:

- Determinación del orden del modelo. Significa establecer cuantos valores de la TGT han de ser conocidos por adelantado para la correcta predicción de uno nuevo.

- Comprobación de la consistencia del modelo, a través de una comparación con los datos originales del horno.

- Establecer la capacidad de predicción, comparando los valores predichos por el modelo con los medidos en el horno.

El sistema utilizado para evaluar estos aspectos consiste en la construcción de once modelos diferentes (en la ec. [1] el rango de $p$ va de 1 a 11). Tras 
ésto, se ha empleado el test de Akaike (14) para determinar cuál de ellos es el más apropiado.

La función de Akaike (AIC) se usa porque en la presente aplicación el efecto del orden del modelo no es demasiado crucial. Solamente es necesario distinguir entre patrones de funcionamiento normales y anormales. En otro caso, sería necesario analizar la potencia de densidad espectral (APSD), cuya forma cambia significativamente para pequeñas modificaciones en el orden del modelo.

La función AIC (15) se define en este caso como:

$$
\operatorname{AIC}(p)=N \cdot \log \left(\sigma^{2}\left(\operatorname{res}_{\mathrm{P}}\right)\right)+2 p
$$

donde:

AIC $=$ Función de Akaike

$p=$ Orden del modelo AR

$N=$ Número de valores de la muestra

$\operatorname{res}_{\mathrm{P}}=$ Resto de orden $p$

\section{RESULTADOS Y DISCUSIÓN}

La TGT se considera más relevante cada día para el estudio del flujo de gas en el interior del horno. Todavía más, es una de las características del horno que puede evaluarse fácilmente, ya que la medida del perfil de temperaturas en el tragante y el análisis continuo de gas en el mismo suministran conjuntos de medidas fiables en cantidad suficiente para ser representativas del comportamiento de la TGT.

En la figura 1 se puede ver el perfil de la TGT, que tiene una morfología adecuada para un modelo autorregresivo. En la figura 2 se muestra la forma de la función de autocorrelación.

Se han estudiado once modelos, y tras calcular los valores de los correspondiente restos, así como los valores de la función de Akaike para cada uno, la elección ha sido el modelo de orden 6 .

Los valores obtenidos mediante dicho modelo se ofrecen en la tabla I.

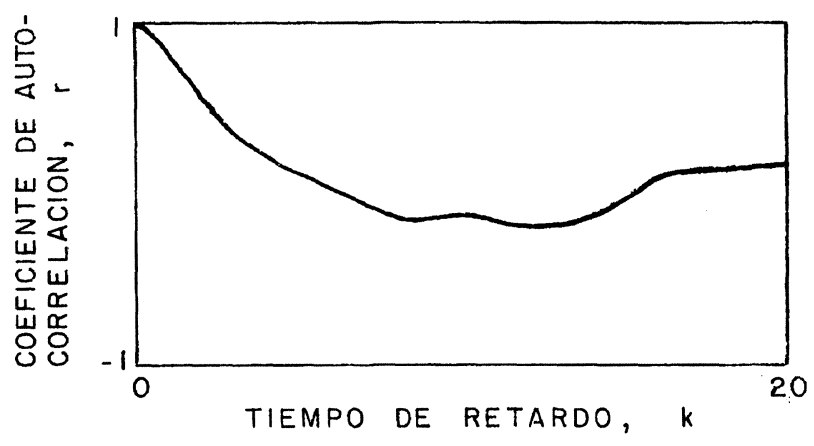

FIG. 2.- Función de autocorrelación de la TGT.

FIG. 2.-Autocorrelation function of the TGT.
TABLA I.- Coeficientes del modelo autorregresivo, $\mathrm{AR}$, de orden 6 para la temperatura de gas en tragante, TGT

TABLE I.- Coefficients of the 6th order autorregresive model, $A R$, for the top gas temperature, TGT

\begin{tabular}{|c|c|c|c|c|c|c|}
\hline$k$ & 1 & 2 & 3 & 4 & 5 & 6 \\
\hline$a(k)$ & 1,104 & $-0,221$ & $-0,235$ & 0,12 & 0,13 & $-0,161$ \\
\hline
\end{tabular}

Para comprobar la consistencia del modelo, se ha efectuado un análisis del resto. El resto es la diferencia existente entre la señal original y la producida por el modelo. La figura 3 muestra el perfil de la señal generada por el modelo de orden 6 , que es muy similar al de la figura 1 para la señal original. La figura 4 muestra el perfil del resto. Su morfología es claramente similar a un ruido blanco, prácticamente aleatorio, con algunos picos pero sin estacionalidades marcadas.

De acuerdo con la ecuación del modelo, este resto ha de ser un ruido blanco si el modelo es correcto. Para establecerlo, se ha estudiado la función de autocorrelación del resto, que, tal y como muestra la figura 5, está suficientemente cercana a una Delta de Dirac, que sería el perfil perfecto para un ruido blanco.

Los análisis anteriores muestran la corrección del modelo en lo que a la reproducción de la señal se refiere. Con respecto a la predicción, en la tabla II se presentan, de forma paralela, los valores predichos por el modelo autorregresivo de orden 6 $(\operatorname{AR6}(t))$ y los medidos en la señal original de la temperatura de gas de tragante (TGT $(t))$.

Como se puede observar en dicha tabla, el modelo permite predecir el comportamiento de la TGT con un error menor del $10 \%$ en los cinco valores siguientes de la variable. En cualquier caso, y

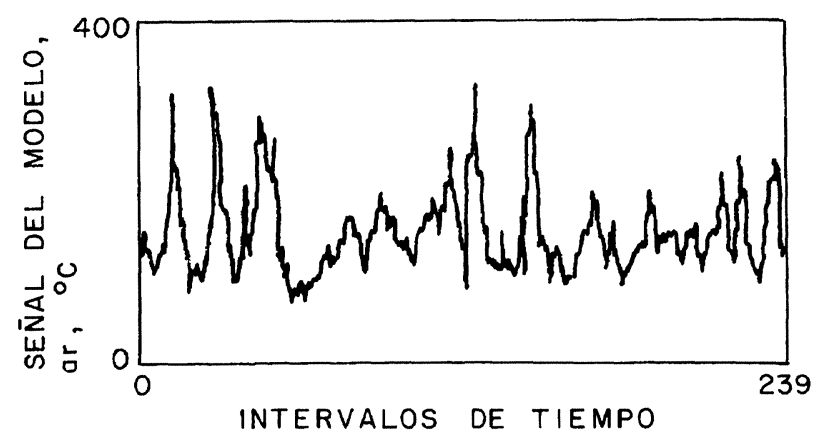

Fig. 3.- Perfil de la señal generada por el modelo AR de orden 6 de la TGT.

FIG. 3.- Profile of the signal generated by the 6th order AR model of the TGT. 


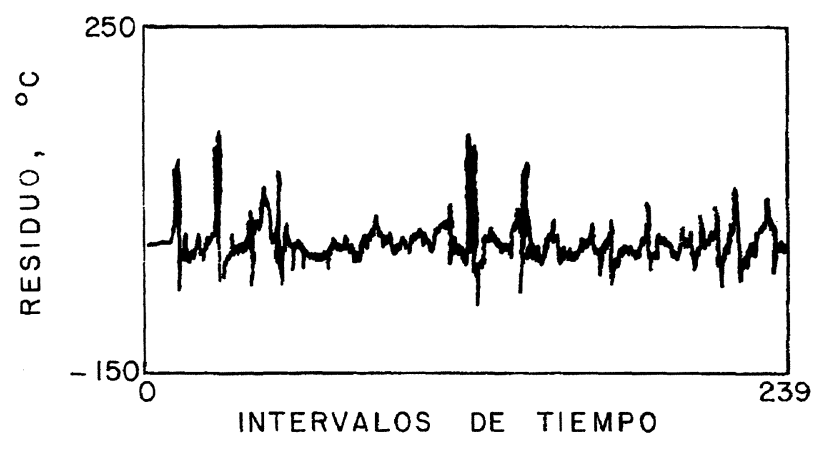

FIG. 4.- Residuo producido por el modelo AR de orden 6 de la TGT.

FIG. 4.- Remainder produced by the 6th order AR model of the TGT.

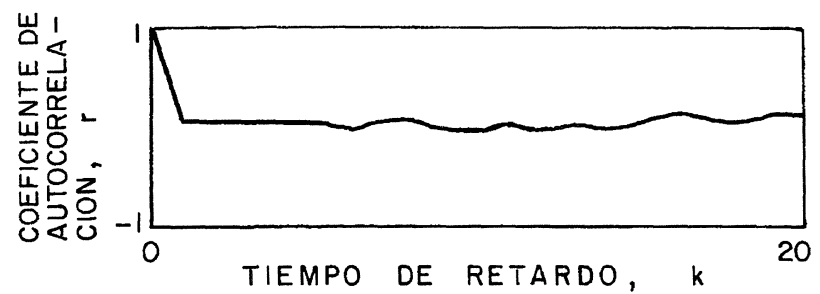

FIG. 5.- Función de autocorrelación del residuo producido por el modelo AR de orden 6 de la TGT.

FIG. 5.- Autocorrelation function of the remainder produced by the 6th order AR model of the TGT.

TABLA II.- Valores generados por el modelo AR de orden 6 en comparación con los datos originales de la TGT, especificando el error producido

TABLE II.- Values obtained through the 6th order AR model compared with the experimental TGT values, showing the produced error

\begin{tabular}{|c|c|c|}
\hline TGT, ${ }^{\circ} \mathrm{C}$ & $\mathrm{AR}(6),{ }^{\circ} \mathrm{C}$ & Error, $\%$ \\
\hline 120 & 123 & 2,5 \\
126 & 122 & 3,2 \\
134 & 127 & 5,2 \\
149 & 137 & 8 \\
145 & 132 & 9 \\
\hline
\end{tabular}

teniendo en cuenta que un error superior al $6 \%$ puede ser problemático, se puede considerar como muy adecuada la predicción para los tres primeros valores, es decir, para períodos de $18 \mathrm{~min}$, que es un resultado altamente satisfactorio para un técnico de horno alto.

\section{CONCLUSIONES}

El perfil de las señales estudiadas presenta una morfología adecuada para su modelización con modelos autorregresivos (AR). Está claro que al variar las muestras los valores de los coeficientes cambiarán, e incluso puede hacerlo el orden del modelo correcto, pero se ha demostrado que esta técnica es suficientemente consistente para su uso en el control del horno alto.

La realización de modelos AR permite predecir el comportamiento de la temperatura de gas en tragante (TGT) con períodos de adelanto de 15-20 min y con un error menor del $6 \%$.

\section{Agradecimiento}

Los autores agradecen a la División de Horno Alto de ENSIDESA, especialmente al Dr. Oscar Rodríguez Yenes, su colaboración como suministradores de los datos experimentales. Este trabajo se ha llevado a cabo dentro del acuerdo núm. 7210AA/934 del Programa de Acero de la Comunidad Europea del Carbón y del Acero (CECA), y del Proyecto CE93/0011, del Programa PGC de la Dirección General de Investigación Científica y Técnica.

\section{REFERENCIAS}

(1) DARTNELl, J. Ironmaking Steelmaking, 5 (1), 1978: 18-24.

(2) Bowness, M.M., Willmers, R.R., PARRATt, J.E. y DaVIES, R.J. Ironmaking Steelmaking, 14 (4), 1987: 150-170.

(3) Asensio, F.J. y Formoso, A. Rev. Metal. Madrid, 9 (3), 1973: 193-199.

(4) Formoso, A., Asensio, F.J., Cubillo, C. y Ruiz, J.C. Rev. Metal. Madrid, 17 (2), 1981: 71-84.

(5) Larrea, M.T., Formoso, A., Sirgado, M., Gutiérrez, A., GÓMEZ, P.P. y CORES, A. Ironmaking Steelmaking, 19 (4), 1992: 311-319.

(6) RENGERSEN, J. Improvement of blast furnace burden quality, ECSC agreement No. 7210-AB/602. Hoogovens, (Países Bajos), abr. 1989.

(7) Committee on Reaction within Blast Furnace. Blast furnace phenomena and modelling. Ed. Elsevier Applied Science. Londres, 1987.

(8) Kanbara, K., Hagwara, T., Shigemi, A., Kondo, S., Kanayama, Y., Wakabayashi, K. e Hiramoto, N. Trans. Iron Steel Inst. Jpn., 17 (7), 1977: 371-380.

(9) MartíneZ, A. Modelo para la automatización del cálculo de las principales características de un horno alto. Tesis Doctoral. Universidad Complutense. Madrid, 1993.

(10) Forrester, J.W. Industrial dynamics. M.I.T. Press. Cambridge, Massachusetts, 1961.

(11) Box, G.E.P. y Jenkins, G.M. Time series analysis. Forecasting and control. Ed. Holden Day. Londres, 1976.

(12) NewLand, D.E. An introduction to random vibrations and spectral analysis. Ed. Longman. Londres, 1975.

(13) RAO, C.R. y Mrtra, S.K. Generalized inverse of matrices and its applications. Ed. John Wiley and Sons. Nueva York, 1971.

(14) AKAIKE, H. IEEE Trans. on auto. contr. AC19, 1974: 716723.

(15) Kitagawa, G. y Akaike, H. Ann. Inst. Statis. Math., (308), 1978: 351-363. 